\title{
Nuclear data uncertainty propagation by the XSUSA method in the HELIOS2 lattice code
}

\author{
Charles Wemple ${ }^{1, a}$ and Winfried Zwermann ${ }^{2}$ \\ 1 Studsvik Scandpower, Inc., 1070 Riverwalk Dr, Suite 150, Idaho Falls, ID 83402, USA \\ 2 Gesellschaft für Anlagen- und Reaktorsicherheit (GRS) gGmbH, Boltzmannstr. 14, 85748 Garching, Germany
}

\begin{abstract}
Uncertainty quantification has been extensively applied to nuclear criticality analyses for many years and has recently begun to be applied to depletion calculations. However, regulatory bodies worldwide are trending toward requiring such analyses for reactor fuel cycle calculations, which also requires uncertainty propagation for isotopics and nuclear reaction rates. XSUSA is a proven methodology for cross section uncertainty propagation based on random sampling of the nuclear data according to covariance data in multigroup representation; HELIOS2 is a lattice code widely used for commercial and research reactor fuel cycle calculations. This work describes a technique to automatically propagate the nuclear data uncertainties via the XSUSA approach through fuel lattice calculations in HELIOS2. Application of the XSUSA methodology in HELIOS2 presented some unusual challenges because of the highly-processed multi-group cross section data used in commercial lattice codes. Currently, uncertainties based on the SCALE 6.1 covariance data file are being used, but the implementation can be adapted to other covariance data in multi-group structure. Pin-cell and assembly depletion calculations, based on models described in the UAM-LWR Phase I and II benchmarks, are performed and uncertainties in multiplication factor, reaction rates, isotope concentrations, and delayedneutron data are calculated. With this extension, it will be possible for HELIOS2 users to propagate nuclear data uncertainties directly from the microscopic cross sections to subsequent core simulations.
\end{abstract}

\section{Introduction}

Uncertainty quantification has been extensively applied to nuclear criticality analyses for many years and has recently begun to be applied to depletion calculations. However, regulatory bodies worldwide are trending toward requiring such analyses for reactor fuel cycle calculations, which also requires uncertainty propagation for isotopics and nuclear reaction rates. To help facilitate cooperation in the development of methods and modelling for uncertainty analyses, the OECD/NEA NSC organized an international expert group for Uncertainty Analysis in Modelling (UAM) in 2005. This expert group has since periodically distributed computational benchmarks for use in development and testing of uncertainty analysis methods, most recently in April 2016.

XSUSA [1] is a proven methodology for cross section uncertainty propagation based on random sampling of the nuclear data according to covariance data in multigroup representation; HELIOS2 [2] is a lattice code widely used for commercial and research reactor fuel cycle calculations. This work describes a technique to automatically propagate the nuclear data uncertainties via the XSUSA approach through fuel lattice calculations in HELIOS2.

\section{Methods}

This section gives a short overview of the implementation of the sampling methodology in HELIOS2, and the underlying data.

\subsection{XSUSA}

The sampling-based uncertainty and sensitivity analysis software XSUSA ("Cross Section Uncertainty and Sensitivity Analysis") has been developed for evaluating the output uncertainties of neutron transport calculations, stand-alone or in combination with depletion or thermohydraulics, which arise from uncertainties in the underlying nuclear data. To this end, a large number of calculations for the problem under consideration are performed with varied nuclear data. The variations of the nuclear data are generated randomly from the given uncertainties and correlations among them as contained in the nuclear covariance matrices. After performing all the calculations, the output quantities of interest are statistically analysed, and their uncertainty ranges are determined. Within XSUSA, the variations are being applied to the cross sections after the resonance self-shielding calculations. This means that it is assumed that the relative uncertainties of the shielded cross sections are identical to those of the unshielded ones, and so-called implicit effects are not taken into account. It has been demonstrated that this approximation only slightly influences the resulting uncertainties of output quantities such as the multiplication factor or reaction rates; examples are given in Ref. [2]. At present, XSUSA is being applied with the set of

\footnotetext{
a e-mail: charles.wemple@studsvik.com
}

(C) The Authors, published by EDP Sciences. This is an Open Access article distributed under the terms of the Creative Commons Attribution License 4.0 (http://creativecommons.org/licenses/by/4.0/). 
covariance data in a 44 energy group representation as contained in the SCALE 6.1 code package [3], which also has been used for the present work. This library contains data for the following fundamental reaction parameters: (n, $\gamma),(n, p),(n, d),(n, t),\left(n,{ }^{3} H e\right),(n, \alpha)$, fission, total $v, \chi$, elastic scattering, inelastic scattering, and (n,2n). Currently, XSUSA is being extended to handle covariance data in other energy group structures, such as the 56group ENDF/B-VII.1 based covariance data set presently shipping with SCALE 6.2.

\subsection{HELIOS2 modifications}

The adaptation of the perturbation vectors from XSUSA to HELIOS2 presented two interesting challenges. First, the 44-group SCALE 6.1 energy structure has very few common bin boundaries with the HELIOS2 library group structures. This required mapping the 44-group bin boundaries into the selected HELIOS2 group structure:

$$
f_{G}=\sum_{g \in G} w_{g} f_{g}
$$

where $g$ denotes the SCALE groups, $G$ the HELIOS2 groups, $f$ the perturbation factors, and $w$ the fraction of the width of group $G$ covered by group $g$. The mapping was performed in lethargy space, to eliminate the distorting effects that can be produced by mapping in energy space.

The second challenge was presented by the highly processed nature of commercial lattice code multi-group cross section libraries. In particular, the HELIOS2 libraries contain cross sections for cumulative quantities, such as absorption and total scattering, whereas the perturbation vectors are defined for the fundamental reaction cross sections and corresponding scattering matrices provided in evaluated data sets. This required defining a set of cross section ratios [4] per isotope to perform a weighted sum of the perturbation vectors for the fundamental reactions, providing the cross section perturbations for the cumulative quantities tabulated in the HELIOS2 library:

$$
f_{i}=\sum_{j \in i} \frac{\sigma_{j}}{\sigma_{i}} f_{j}
$$

where $i$ denotes the cumulative reaction type, $j$ the fundamental reaction types, and $f$ the perturbation factors. The total scattering is production based, so the summation counts the $(n, 2 n)$ contribution twice, with the absorption cross section reduced by $\sigma_{n, 2 n}$ to preserve neutron balance. In the scattering matrices, the perturbation factors corresponding to the initial group are applied to each matrix element. The ratios are compiled as a supplementary data set to be processed once per case.

The resulting cross section perturbations in HELIOS2 were applied to the individual isotopic cross sections before the final assembly of the macroscopic cross sections for the transport solution. For depletion calculations, a set of perturbations was applied at each step through the entire depletion path. In addition to the perturbed cases, the base (unperturbed) calculation was also performed, so that a set of 1000 perturbations would result in 1001 full depletion calculations. The case generation was automated for ease of use and can be divided into multiple subsets, to speed computation.
Table 1. Model parameters for the UAM TMI-1 PWR pin-cell.

\begin{tabular}{|l|c|}
\hline Parameter & Value \\
\hline Unit cell pitch, mm & 14.427 \\
\hline Fuel pellet diameter, mm & 9.391 \\
\hline Fuel density, g/cm ${ }^{3}$ & 10.283 \\
\hline Clad outer diameter, mm & 10.928 \\
\hline Clad thickness, mm & 0.673 \\
\hline Reactor power, MW & 2772 \\
\hline EOC burnup, GWd/t & 61.28 \\
\hline Shutdown cooling time, yrs & 100 \\
\hline
\end{tabular}

Table 2. BOC and EOC macroscopic XS and their relative uncertainties (\%) for UAM TMI-1 PWR pin-cell case.

\begin{tabular}{|l|l|l|l|l|}
\hline \multirow{2}{*}{ Value } & \multicolumn{2}{|c|}{ BOC } & \multicolumn{2}{c|}{ EOC } \\
\cline { 2 - 5 } & Result & Uncert & Result & Uncert \\
\hline $\mathrm{k}_{\text {eff }}$ & 1.41274 & 0.4967 & 0.89421 & 0.7667 \\
\hline $\mathrm{D}$ (gr. 1) & 1.078 & 1.1773 & 1.082 & 1.1690 \\
\hline $\mathrm{D}$ (gr. 2) & $3.321 \mathrm{E}-01$ & 0.1991 & $3.265 \mathrm{E}-01$ & 0.2175 \\
\hline$\Sigma_{\mathrm{a}}$ (gr. 1) & $1.129 \mathrm{E}-02$ & 0.9614 & $1.274 \mathrm{E}-02$ & 1.0798 \\
\hline$\Sigma_{\mathrm{a}}$ (gr. 2) & $1.157 \mathrm{E}-01$ & 0.2361 & $1.178 \mathrm{E}-01$ & 1.4582 \\
\hline$\nu \Sigma_{\mathrm{f}}$ (gr. 1) & $9.690 \mathrm{E}-03$ & 0.5026 & $5.404 \mathrm{E}-03$ & 1.6274 \\
\hline$\nu \Sigma_{\mathrm{f}}$ (gr. 2) & $2.101 \mathrm{E}-01$ & 0.4411 & $1.562 \mathrm{E}-01$ & 1.9303 \\
\hline$\Sigma_{\mathrm{f}}$ (gr. 1) & $3.843 \mathrm{E}-03$ & 0.3670 & $1.953 \mathrm{E}-03$ & 1.5058 \\
\hline$\Sigma_{\mathrm{f}}$ (gr. 2) & $8.620 \mathrm{E}-02$ & 0.3244 & $5.619 \mathrm{E}-02$ & 1.7867 \\
\hline$\Sigma_{\mathrm{s} 0}$ (gr. 1) & $5.337 \mathrm{E}-01$ & 0.9614 & $5.300 \mathrm{E}-01$ & 0.9399 \\
\hline$\Sigma_{\mathrm{s} 0}$ (gr. 2) & 1.222 & 0.1470 & 1.238 & 0.1461 \\
\hline
\end{tabular}

\section{Calculations and results}

Application cases from the Benchmark for Uncertainty Analysis in Modelling (UAM) for Design, Operation and Safety Analysis of LWRs have been chosen; model specifications and output requests have been extracted from the corresponding specifications [5,6]. For all calculations, perturbation vectors of length 1000 were generated by XSUSA.

\subsection{Pin-cell calculations}

Initial pin-cell calculations were performed with the UAM TMI-1 pin-cell model [5]. The unit cell has dimensions as shown in Table 1, with $\mathrm{UO}_{2}$ fuel at $4.85 \mathrm{w} / \mathrm{o}{ }^{235} \mathrm{U}$ and hot full power conditions $\left(\mathrm{T}_{\text {fuel }}=900 \mathrm{~K}, \mathrm{~T}_{\text {clad }}=600 \mathrm{~K}\right.$, $\mathrm{T}_{\text {cool }}=562 \mathrm{~K}$ ). Requested results from the benchmark included $\mathrm{k}_{\mathrm{eff}}$, two-group macroscopic cross sections, and isotope concentrations, with accompanying uncertainties. Results were requested at several burnup steps and shutdown cooling times.

Results for $\mathrm{k}_{\mathrm{eff}}$ and two-group macroscopic cross sections at BOC and EOC are shown in Table 2. The calculated uncertainties are comparable to previously compiled results for the pin-cell benchmark [7] and the EOC results clearly show the effect of the larger variances associated with the higher actinides.

\subsection{Assembly calculations}

Full assembly calculations were performed with the UAM TMI-1 PWR assembly model [6]. The assembly layout is described by Fig. 1 and Table 3; depletion was performed to $60 \mathrm{GWd} / \mathrm{t}$ at hot full power conditions with a constant $900 \mathrm{ppm}$ soluble boron in the coolant. The control rod calculations were performed without 


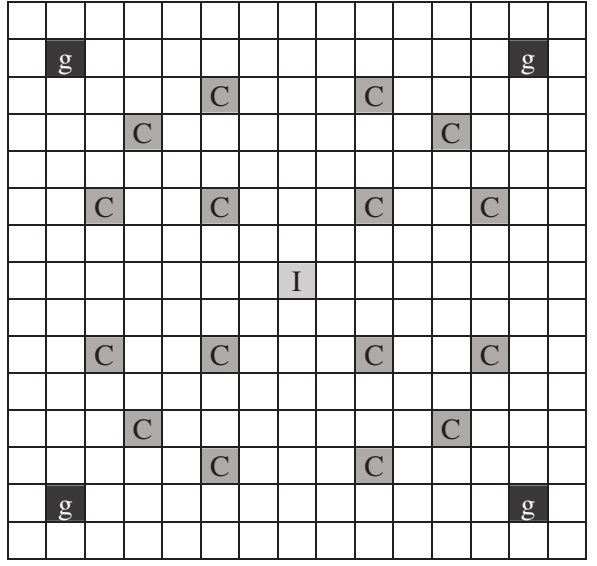

Figure 1. UAM TMI-1 PWR assembly design.

Table 3. Rod type descriptions for TMI-1 assembly (Fig. 1).

\begin{tabular}{|l|l|}
\hline Marker & Rod type \\
\hline & Fuel $-4.12 \%{ }^{235} \mathrm{U}$ \\
\hline $\mathrm{g}$ & Fuel $-4.12 \%{ }^{235} \mathrm{U}, 2.0 \% \mathrm{Gd}_{2} \mathrm{O}_{3}$ \\
\hline $\mathrm{C}$ & CR guide tube \\
\hline $\mathrm{I}$ & Instrument tube \\
\hline
\end{tabular}

Table 4. Macroscopic two-group data and relative uncertainties (\%) for UAM TMI-1 assembly case at BOC and $60 \mathrm{GWd} / \mathrm{t}$.

\begin{tabular}{|c|c|c|c|c|}
\hline \multirow{2}{*}{ Value } & \multicolumn{2}{|c|}{ BOC } & \multicolumn{2}{c|}{60 GWd/t } \\
\cline { 2 - 5 } & Result & Uncert & \multicolumn{1}{|c|}{ Result } & Uncert \\
\hline $\mathrm{k}_{\text {eff }}$ & 1.30160 & 0.4752 & 0.84446 & 0.7749 \\
\hline $\mathrm{D}$ (gr. 1) & 1.088 & 1.1610 & 1.092 & 1.1495 \\
\hline $\mathrm{D}$ (gr. 2) & $3.181 \mathrm{E}-01$ & 0.1912 & $3.147 \mathrm{E}-01$ & 0.2031 \\
\hline$\Sigma_{\mathrm{a}}$ (gr. 1) & $1.071 \mathrm{E}-02$ & 0.9358 & $1.199 \mathrm{E}-02$ & 1.0798 \\
\hline$\Sigma_{\mathrm{a}}$ (gr. 2) & $1.166 \mathrm{E}-01$ & 0.2089 & $1.104 \mathrm{E}-01$ & 1.4582 \\
\hline$v \Sigma_{\mathrm{f}}$ (gr. 1) & $8.9868 \mathrm{E}-03$ & 0.5086 & $4.896 \mathrm{E}-03$ & 1.6274 \\
\hline$\nu \Sigma_{\mathrm{f}}$ (gr. 2) & $1.861 \mathrm{E}-01$ & 0.4408 & $1.306 \mathrm{E}-01$ & 1.9303 \\
\hline$\Sigma_{\mathrm{f}}$ (gr. 1) & $3.552 \mathrm{E}-03$ & 0.3619 & $1.771 \mathrm{E}-03$ & 1.5058 \\
\hline$\Sigma_{\mathrm{f}}$ (gr. 2) & $7.637 \mathrm{E}-02$ & 0.3241 & $4.709 \mathrm{E}-02$ & 1.7867 \\
\hline$\Sigma_{\mathrm{s} 0 \text { (gr. 1) }}$ & $5.424 \mathrm{E}-01$ & 0.9347 & $5.390 \mathrm{E}-01$ & 0.9399 \\
\hline$\Sigma_{\mathrm{s} 0 \text { (gr. 2) }}$ & 1.285 & 0.1378 & 1.299 & 0.1461 \\
\hline $1 / \mathrm{v}$ (gr. 1) & $1.231 \mathrm{E}-02$ & 1.2423 & $1.147 \mathrm{E}-02$ & 1.2348 \\
\hline $1 / \mathrm{v}$ (gr. 2) & $5.192 \mathrm{E}-01$ & 0.0348 & $5.437 \mathrm{E}-01$ & 0.1370 \\
\hline ADF (gr. 1) & 1.022 & 0.0318 & 1.031 & 0.0616 \\
\hline ADF (gr. 2) & $7.816 \mathrm{E}-01$ & 0.0507 & $7.617 \mathrm{E}-01$ & 0.2796 \\
\hline
\end{tabular}

soluble boron. Requested results from the depletion benchmark included $\mathrm{k}_{\mathrm{eff}}$, two-group macroscopic cross sections and assembly discontinuity factors, inverse neutron velocity, effective delayed neutron fractions, pin burnup distributions, and EOC isotope concentrations, with accompanying uncertainties. Results were requested at several burnup steps through the depletion calculation.

\subsubsection{Depletion calculations}

The two-group data (macroscopic XS, inverse velocities, ADF) from the depletion calculation are shown in Table 4, and the delayed neutron fractions in Table 5.

The uncertainties are comparable with other compilations of results for this benchmark [7]. With respect to the uncertainties of the delayed neutron fractions, note that the SCALE 6.1 covariance data do not contain uncertainties of the average number of delayed neutrons per fission $(v)$ separately, but only for the total $v$. However, the delayed
Table 5. Effective delayed neutron fractions and their relative uncertainties (\%) for UAM TMI-1 PWR assembly case at BOC and $60 \mathrm{GWd} / \mathrm{t}$.

\begin{tabular}{|c|c|c|c|c|}
\hline Delayed & \multicolumn{2}{|c|}{ BOC } & \multicolumn{2}{c|}{ 60 GWd/t } \\
\cline { 2 - 5 } Group & Fraction & Uncert & Fraction & Uncert \\
\hline 1 & $2.049 \mathrm{E}-04$ & 0.0691 & $1.156 \mathrm{E}-04$ & 0.3925 \\
\hline 2 & $1.123 \mathrm{E}-03$ & 0.1707 & $9.235 \mathrm{E}-04$ & 0.4756 \\
\hline 3 & $1.105 \mathrm{E}-03$ & 0.2332 & $7.624 \mathrm{E}-04$ & 0.7887 \\
\hline 4 & $3.230 \mathrm{E}-03$ & 0.3453 & $2.076 \mathrm{E}-03$ & 1.1367 \\
\hline 5 & $1.034 \mathrm{E}-03$ & 0.7436 & $8.291 \mathrm{E}-04$ & 1.5011 \\
\hline 6 & $3.460 \mathrm{E}-04$ & 0.5232 & $2.391 \mathrm{E}-04$ & 1.4125 \\
\hline
\end{tabular}

Table 6. Nuclide concentrations (atoms/barn-cm) and relative uncertainties (\%) for the UAM TMI-1 PWR assembly case at EOC.

\begin{tabular}{|l|c|c|}
\hline Isotope & Concentration & Uncertainty \\
\hline $\mathrm{U}-235$ & $1.60 \mathrm{E}-04$ & 2.1533 \\
\hline $\mathrm{U}-236$ & $1.58 \mathrm{E}-04$ & 1.4460 \\
\hline $\mathrm{U}-238$ & $2.07 \mathrm{E}-02$ & 0.0364 \\
\hline $\mathrm{Pu}-239$ & $1.36 \mathrm{E}-04$ & 2.1696 \\
\hline $\mathrm{Pu}-240$ & $6.75 \mathrm{E}-05$ & 2.4003 \\
\hline $\mathrm{Pu}-241$ & $4.19 \mathrm{E}-05$ & 1.9089 \\
\hline $\mathrm{Pu}-242$ & $2.39 \mathrm{E}-05$ & 3.6290 \\
\hline $\mathrm{Am}-241$ & $1.69 \mathrm{E}-06$ & 3.4094 \\
\hline $\mathrm{Am}-243$ & $6.58 \mathrm{E}-06$ & 7.3024 \\
\hline $\mathrm{Cm}-242$ & $6.92 \mathrm{E}-07$ & 1.6264 \\
\hline $\mathrm{Cm}-244$ & $3.36 \mathrm{E}-06$ & 7.9305 \\
\hline $\mathrm{Mo}-95$ & $6.90 \mathrm{E}-05$ & 0.3587 \\
\hline Tc-99 & $7.15 \mathrm{E}-05$ & 0.4695 \\
\hline $\mathrm{Ru}-101$ & $7.29 \mathrm{E}-05$ & 0.6678 \\
\hline $\mathrm{Ag}-109$ & $6.84 \mathrm{E}-06$ & 1.3663 \\
\hline $\mathrm{I}-129$ & $1.15 \mathrm{E}-05$ & 0.3654 \\
\hline $\mathrm{Xe}-135$ & $7.56 \mathrm{E}-09$ & 3.4407 \\
\hline $\mathrm{Cs}-137$ & $8.45 \mathrm{E}-05$ & 0.0339 \\
\hline $\mathrm{Ce}-144$ & $1.43 \mathrm{E}-05$ & 0.0806 \\
\hline $\mathrm{Nd}-148$ & $2.42 \mathrm{E}-05$ & 0.3975 \\
\hline Sm-149 & $9.55 \mathrm{E}-08$ & 2.7349 \\
\hline Eu-155 & $5.62 \mathrm{E}-07$ & 5.4565 \\
\hline $\mathrm{Gd}-158$ & $1.44 \mathrm{E}-06$ & 4.8844 \\
\hline
\end{tabular}

$v$ uncertainties may have a significant influence on the delayed neutron fraction uncertainties [8], such that the values given in Table 5 must be considered too low.

Table 6 provides the nuclide concentrations and associated relative uncertainties $(\%)$ for selected isotopes at EOC. In addition to the factors already considered, the uncertainties of the nuclide concentrations may also be sensitive to fission yield uncertainties. This mainly applies to fission product concentrations; actinide concentrations are primarily influenced by the neutron cross section uncertainties [1]. Although fission yield uncertainties are available in the ENDF files, they do not contain correlations among them. There are indications that such correlations may have a significant influence on the nuclide concentration uncertainties of most fission products. There are presently several competing methods for determining these correlations, and no method has yet achieved wide consensus. Therefore, for the time being, the inclusion of fission yield uncertainties in HELIOS2/XSUSA analyses is postponed until widely accepted covariance data are available. 
Table 7. HFP and HZP, controlled and uncontrolled eigenvalue results and relative uncertainties (\%) for UAM TMI-1 PWR assembly case at BOC.

\begin{tabular}{|l|l|l|}
\hline Case & $\mathbf{k}_{\text {eff }}$ & Uncert \\
\hline HFP uncontrolled & 1.40143 & 0.4746 \\
\hline HFP controlled & 1.06701 & 0.5076 \\
\hline HZP uncontrolled & 1.41287 & 0.4694 \\
\hline HZP controlled & 1.07591 & 0.5021 \\
\hline
\end{tabular}

\subsubsection{Control rod calculations}

A series of controlled and uncontrolled assembly calculations were performed at HFP and HZP conditions at BOC, using the UAM TMI-1 PWR assembly case [5] model parameters. The requested results included $\mathrm{k}_{\mathrm{eff}}$ and pin power maps, with the associated uncertainties. The $\mathrm{k}_{\mathrm{eff}}$ and uncertainty results of these calculations are shown in Table 7.

\section{Summary and future work}

The sampling-based uncertainty and sensitivity analysis software XSUSA and the lattice transport code HELIOS2 have been successfully coupled to produce a system capable of automatically propagating nuclear data uncertainties through lattice depletion calculations. A series of initial validation calculations have been performed, using pincell and assembly models defined within the UAM-LWR benchmark series. The results produced are comparable to previously compiled uncertainties in the literature, demonstrating the proper functioning of the coupled code system.

As next steps, it is planned to perform a comprehensive validation of the HELIOS2/XSUSA code system with criticality and depletion calculations for PWR, BWR, and VVER pin cells and fuel assemblies in the framework of the UAM-LWR Benchmark; the results are considered as benchmark contributions. With the few-group macroscopic fuel assembly cross sections from such calculations, analyses with a core simulator will be performed. The extensions to XSUSA currently in progress, namely the inclusion of individual uncertainties for delayed $v$, as well as the use of covariance data in arbitrary energy group representation, are also planned to be included. Finally,
HELIOS2 customers will be provided with the extended code version to be able to perform uncertainty analyses with respect to the uncertainties of the underlying nuclear data.

The work of GRS is supported by the German Federal Ministry for Economic Affairs and Energy.

\section{References}

[1] F. Bostelmann, et al., "Sampling-Based Nuclear Data Uncertainty Analysis in Criticality and Depletion Calculations," Proceedings of ANS MC2015, Nashville, TN, USA, 19-23 April (2015)

[2] C.A. Wemple, et al., "Recent Advances in the HELIOS-2 Lattice Physics Code,"Proceedings of PHYSOR 2008, Interlaken, Switzerland, 14-19 September (2008)

[3] "SCALE: A Comprehensive Modeling and Simulation Suite for Nuclear Safety Analysis and Design," ORNL/TM-2005/39, Version 6.1 (2011)

[4] W. Wieselquist, A. Vasiliev, H. Ferroukhi, "Nuclear Data Uncertainty Propagation in a Lattice Physics Code Using Stochastic Sampling," Proceedings of PHYSOR 2012, Knoxville TN USA, 15-20 April (2012)

[5] K. Ivanov, et al., "Benchmarks for Uncertainty Analysis in Modelling (UAM) for the Design, Operation and Safety Analysis of LWRs, Vol. I," NEA/NSC/DOC(2013), available from OECD/NEA Data Bank

[6] T. Blyth, et al., "Benchmarks for Uncertainty Analysis in Modelling (UAM) for the Design, Operation and Safety Analysis of LWRs, Vol. II," NEA/NSC/DOC(2016), available from OECD/NEA Data Bank

[7] R.N. Bratton, M. Avramova, K. Ivanov, "OECD/NEA Benchmark for Uncertainty Analysis in Modeling (UAM) for LWRs - Summary and Discussion of Neutronics Cases (Phase I)," Nucl. Eng. Tech. 46(3), 313-342 (2014)

[8] A. Aures, et al., "Uncertainty in the Delayed Neutron Fraction in Fuel Assembly Depletion Calculations," Proceedings of ND2016, Bruges, Belgium, 10-16 Sep. (2016) 